\title{
Low Doses of Rifampicin Used in New Tuberculosis Patients Correlated to Increased Frequency of Rifampicin-Resistance and Poorer Treatment Outcomes
}

\author{
Ling Chen ${ }^{*}$, Jian $\mathrm{Du}^{2 *}$, Liang $\mathrm{Li}^{2}$, Qi Li², Qiu Zhong3, Yanyong Fu4, Bo Li5, Minggui Lin6, \\ Liping $\mathrm{Ma}^{7}$, Youlun $\mathrm{Li}^{8}$, Xiaomeng Wang ${ }^{9}$, Yan $\mathrm{Ma}^{2}$, Xiaoying Jiang ${ }^{2}$, Xiaoyou Chen ${ }^{2}$, \\ Qiping Ge², Li Xie², Xiqin Han², Zhaogang Sun², Guanglu Jiang2, Hong Zhang,,10\#, \\ Weiwei Gao"\# \\ ${ }^{1}$ Affiliated Hospital of Zunyi Medical College, Zunyi, China \\ ${ }^{2}$ Beijing Chest Hospital/Beijing Institute of Tuberculosis and Thoracic Tumor, Beijing, China \\ ${ }^{3}$ Guangdong Center for Tuberculosis Control, Guangzhou, China \\ ${ }^{4}$ Tianjin Center for Tuberculosis Control, Tianjin, China \\ ${ }^{5}$ Beijing Institute for Tuberculosis Control, Beijing, China \\ ${ }^{6}$ 309th Hospital of PLA, Beijing, China \\ ${ }^{7}$ Henan Center for Disease Control, Zhengzhou, China \\ ${ }^{8}$ First Affiliated Hospital, Chongqing Medical University, Chongqing, China \\ ${ }^{9}$ Zhejiang Center for Disease Control, Hangzhou, China \\ ${ }^{10}$ Z-BioMed, Inc., Rockville, MD, USA \\ Email: "hzhang@zbiomed.com, "gwwjys@sina.com
}

Received 22 April 2015; accepted 30 May 2015; published 2 June 2015

Copyright (C) 2015 by authors and Scientific Research Publishing Inc.

This work is licensed under the Creative Commons Attribution International License (CC BY).

http://creativecommons.org/licenses/by/4.0/

(c) (i) Open Access

\section{Abstract}

The prognosis of patients with previously treated tuberculosis (TB) was suggested to be dependent on whether the initial treatment was in compliance with the established guidelines. The aim of this retrospective multicenter study was to determine the proportion of new TB patients who received standard doses of rifampicin in multiple provinces of China, and the relationship between low doses of rifampicin and frequency of rifampicin-resistance as well as treatment out-

\footnotetext{
${ }^{*}$ These authors contributed equally to this work.

\#Corresponding authors.
}

How to cite this paper: Chen, L., et al. (2015) Low Doses of Rifampicin Used in New Tuberculosis Patients Correlated to Increased Frequency of Rifampicin-Resistance and Poorer Treatment Outcomes. Open Journal of Medical Microbiology, 5, 7684. http://dx.doi.org/10.4236/ojmm.2015.52009 
comes. A total of 713 new TB patients were treated with either once-daily dose of bulk anti-TB drugs (group I) or every other day combination blister packs of anti-TB drugs containing rifampicin (group II) at more than 30 TB treatment centers/hospitals in China. Treatment history, therapeutic doses of rifampicin, and information about patients were extracted from their medical records and analyzed, and rifampicin-resistance of isolates collected from patients following the treatment as well as treatment outcomes were compared between two treatment groups. Among 522 patients in treatment group I, $154(29.5 \%)$ received standard and $363(69.5 \%)$ received low doses of rifampicin; 238 (45.6\%) isolates were rifampicin-resistant, and $243(46.6 \%)$ were successfully treated. Among 191 patients in treatment group II, 175 (91.6\%) received standard and $15(7.9 \%)$ received low doses of rifampicin; $72(37.7 \%)$ isolates were rifampicin-resistant, and $105(55 \%)$ were successfully treated. When patients who received low doses of rifampicin were compared to others within the same treatment group, increased rates for rifampicin-resistance and treatment failure were observed. Results from this study showed that most new TB patients in treatment group I (69.5\%) received low doses of rifampicin, and their treatment outcomes were worse than those in treatment group II, indicating that low doses of rifampicin used for the initial treatment of new TB patients were correlated to increased frequency of rifampicin-resistance and poorer treatment outcomes.

\section{Keywords}

Mycobacterium tuberculosis, Rifampicin, Therapeutic Doses, Drug Resistance, Treatment Outcomes

\section{Introduction}

Tuberculosis (TB) is one of the leading infectious diseases in the world. An estimated 9.0 million people developed TB in 2013 and 0.48 million of them were multidrug-resistant (MDR)-TB [1]. To improve treatment outcomes and prevent the transmission of drug-resistant Mycobacterial tuberculosis (M.tb), it is crucial to treat new cases of pulmonary TB with standard doses of four first-line anti-TB drugs (isoniazid, rifampicin, ethambutol, and pyrazinamide) [2] [3]. Each of the four first-line anti-TB drugs plays an important role in the treatment of TB and rifampicin is one of the most effective anti-TB drugs [3].

Previous studies suggested that the prognosis of patients with previously treated pulmonary TB was dependent on whether or not the initial treatment of new cases was in compliance with the established guidelines [2][5], and the risk of developing drug-resistant TB and MDR-TB was increased dramatically in patients who received inadequate treatments [6]. Previous studies also indicated that one of the factors causing drug resistance could be the insufficient dose of drugs given to TB patients [7], that the plasma concentration of rifampicin was related to the body weight of patients, and that increased body weight could reduce the plasma concentration of rifampicin [8] [9]. When high-verse standard-doses of rifampicin used in Indonesia patients with pulmonary TB were compared, the mean peak plasma concentration of rifampicin in the high-dose (600 $\mathrm{mg}$ ) group was found to be higher than that in the standard-dose $(450 \mathrm{mg}$ ) group and the difference was statistically significant, whereas the enzyme activity of glutamic-pyruvic transaminase between two groups had no significant difference [10]. Recently published studies also showed that bioavailability of rifampicin in four out of five fixed-dose combinations used in China was not within the acceptable therapeutic range of $80 \%-125 \%$ [11], that three two-drug fixed-dose combinations used in a clinical trial in China displayed inferior rifampicin bioavailability compared with the reference drug [12], and that increasing the rifampicin concentration could inhibit the growth of rifampicin-resistant $M$. tuberculosis in vitro [13].

In this retrospective multicenter study, we aimed to determine the proportion of new TB patients who received standard doses of rifampicin in multiple provinces of China and the relationship between low doses of rifampicin used for the initial treatment of new TB patients and frequency of rifampicin-resistance as well as treatment outcomes. 


\section{Methods}

\subsection{Setting and Study Population}

The Medical Ethics Committee at Beijing Chest Hospital approved this study before the study began, and written informed consent forms were signed by participants for their clinical records to be used in this study. Patients diagnosed as sputum smear positive (acid-fast bacilli staining) and culture positive pulmonary TB were treated previously with bulk drugs or combination blister packs of anti-TB drugs at more than 30 TB prevention and treatment centers/hospitals in multiple provinces of China between July 2009 and July 2011. The selection criteria of patients included those who were diagnosed as active pulmonary TB and treated previously. Patients were ineligible for this retrospective multicenter study if they had non-TB mycobacterial lung diseases, combined extrapulmonary TB, combined severe diseases in heart, liver and kidney, malnutrition, human immunodeficiency virus (HIV), diabetes complications, pneumoconiosis, blood disorders, digestive diseases, etc. Patients were also excluded if their records were incomplete.

For the initial treatment of new TB patients, physicians at specialized TB hospitals usually prescribe oncedaily doses of bulk anti-TB drugs including rifampicin $(300-750 \mathrm{mg})$ individually to patients according to their different circumstances (group I). Whereas, health care providers at TB prevention and treatment centers (or local sites of the Chinese CDC) generally use combination blister packs of anti-TB drugs containing $600 \mathrm{mg}$ of rifampicin as the intermittent therapy every other day for new TB patients (group II). Patients in both groups received an initial phase of two-month oral regimens of four first-line anti-TB drugs followed by a continuation phase of about four months using isoniazid and rifampicin.

\subsection{Data Collection}

Staff involved in collection of data from patients, culture of sputum samples collected from TB patients in two treatment groups following the treatment on Löwenstein-Jensen (L-J) solid media (purchased from the same supplier), drug susceptibility testing (DST) on collected isolates with M. tuberculosis strain H37Rv as the control, and submission of data online at each center were trained by experts using the same training materials and standard operation protocols.

A standard web-based questionnaire was created specifically for this study to extract a variety of data about TB patients who were treated between July 2009 and July 2011 in two treatment groups at more than 30 TB prevention and treatment centers/hospitals in multiple provinces of China. The data including previous treatment history, therapeutic doses of rifampicin used in the initial treatment, DST results against rifampicin for isolates collected from patients following the treatment, treatment outcomes, plus gender, age, and body weight of patients at the time of the initial treatment were extracted from patients' medical records at each participating center or hospital. After collection at each participating center, data were uploaded immediately to the main server located in Beijing Chest Hospital. Completed reports were reviewed and verified by the project manager, and selected for this study if they met the inclusion criteria.

\subsection{Data Analysis}

According to the established guidelines [2]-[5], standard doses of rifampicin should be: $450 \mathrm{mg}$ per day for patients weighting $<50 \mathrm{~kg}$ and $600 \mathrm{mg}$ per day for patients weighting $\geq 50 \mathrm{~kg}$ with $600 \mathrm{mg}$ as the maximal daily dose for patients in treatment group I; and $600 \mathrm{mg}$ per day for patients in treatment group II who weight either $<50 \mathrm{~kg}$ or $\geq 50 \mathrm{~kg}$. Collected data were analyzed and compared to determine what proportions of patients in each group were in compliance with the standard doses of rifampicin and to find the correlation between low doses of rifampicin used for the initial treatment of new TB patients and frequency of rifampicin-resistance as well as treatment outcomes. Data management and statistical analysis were performed using the SPSS 17.0 software. The Fisher's Exact Test Calculator for two $\times$ two Contingency Tables

(http://research.microsoft.com/en-us/um/redmond/projects/mscompbio/fisherexacttest/) and Pearson's chi-square test were used to analyze and compare categorical variables, and $P<0.05$ was considered statistically significant.

\section{Results}

A total of 713 patients with previously treated smear positive pulmonary M. tuberculosis were selected for this 
retrospective multicentre study (Table 1). The number of male patients (527) was significantly higher than that of female patients (186), which was consistent with the 2010 National Epidemiological Survey of China [14]. The age ranges of patients were from 12 to 87 years old and 673 of them (94.4\%) were between the age of 20 and 59; the range of body weight was from $32.5 \mathrm{~kg}$ to $115 \mathrm{~kg}$ with the average body weight of $56.5 \mathrm{~kg}$, and 475 of them (66.6\%) were between body weight of 50 and $70 \mathrm{~kg}$ (Table 1). Out of 522 patients in treatment group I, $154(29.5 \%)$ received standard doses of rifampicin, 363 (69.5\%) received low doses of rifampicin, and five (1.0\%) were overdosed (Table 2). As shown in Table 2, 74.6\% of male patients received low doses of rifampicin which was significantly higher than that of female patients (55.7\%). There was a significant difference in proportion of patients received standard doses of rifampicin between patients weighing below $50 \mathrm{~kg}(90.9 \%)$ and equal or above $50 \mathrm{~kg}(13.1 \%)$. Out of 191 patients in treatment group II, 175 (91.6\%) received standard doses and 15 (7.9\%) received low doses of rifampicin, and only one (0.5\%) received high dose of rifampicin (Table 3). There was no significant difference in proportion of patients received standard doses of rifampicin among different age groups from 20 to 70 and older $(P=0.462)$ and patients with different body weight $(P=$ $0.336)$.

To determine whether low doses of rifampicin used in the initial treatment of new TB patients were correlated to increased frequency of rifampicin-resistance and poorer treatment outcomes in previously treated TB patients, we compared the number of patients who were treated with low doses of rifampicin, the frequency of rifampicin-resistance and treatment outcomes between two treatment groups following the treatment. As indicated in Table 4, 69.5\% of patients (363/522) in treatment group I, and 7.9\% of patients (15/191) in therapeutic group II were initially treated with low doses of rifampicin; and the difference between two groups was statistically significant $(P<0.0001)$.

Results from drug susceptibility testing (DST) conducted on M.tb cultures collected following the treatment showed that $45.6 \%$ of clinical isolates (238/522) collected from TB patients in treatment group I and $37.7 \%$ of clinical isolates (72/191) collected from TB patients in treatment group II were resistant to rifampicin, and the difference between two groups was statistically significant $(P<0.0304)$. We also observed that rifampicin-

Table 1. Demographic characteristics of study participants in two treatment groups.

\begin{tabular}{|c|c|c|c|}
\hline Characteristics & Patient no. (\%) & Group I I $\left.^{\mathrm{a}} \%\right)$ & Group II ${ }^{\mathbf{b}}(\%)$ \\
\hline \multicolumn{4}{|l|}{ Gender } \\
\hline Male & 527 (73.9) & $382(73.2)$ & 145 (75.9) \\
\hline Female & $186(26.1)$ & $140(26.8)$ & $46(24.1)$ \\
\hline \multicolumn{4}{|l|}{ Age group (years) } \\
\hline $12-19$ & $20(2.8)$ & $17(3.3)$ & $3(1.6)$ \\
\hline $20-29$ & 139 (19.5) & $100(19.2)$ & 39 (20.4) \\
\hline $30-39$ & $161(22.6)$ & $117(22.4)$ & $44(23.0)$ \\
\hline $40-49$ & $176(24.7)$ & $132(25.3)$ & $44(23.0)$ \\
\hline $50-59$ & 139 (19.5) & $96(18.4)$ & $43(22.5)$ \\
\hline $60-69$ & $58(8.1)$ & $45(8.6)$ & $13(6.8)$ \\
\hline$>70$ & $20(2.8)$ & $15(2.9)$ & $5(2.6)$ \\
\hline \multicolumn{4}{|l|}{ Weight (kg) } \\
\hline$<50$ & $164(23.0)$ & $110(21.1)$ & $54(28.3)$ \\
\hline $50-70$ & 475 (66.6) & 347 (66.5) & $128(67.0)$ \\
\hline$>70$ & $74(10.4)$ & 65 (12.5) & $9(4.7)$ \\
\hline Total & $713(100)$ & $522(73.2)$ & $191(26.8)$ \\
\hline
\end{tabular}

${ }^{\mathrm{a}}$ Group I, rifampicin once daily therapy doses ranging from $300 \mathrm{mg}$ to $750 \mathrm{mg}$; ${ }^{\mathrm{b}}$ Group II, intermittent therapy every other day with combination blister packs of anti-TB drugs containing $600 \mathrm{mg}$ of rifampicin. 
Table 2. Number and proportion of TB patients treated with different doses of rifampicin in Group I.

\begin{tabular}{|c|c|c|c|c|}
\hline & \multirow{2}{*}{ Patient no. } & \multicolumn{3}{|c|}{ Number (\%) of patients treated with } \\
\hline & & Standard doses & Low doses & High doses \\
\hline \multicolumn{5}{|l|}{ Gender } \\
\hline Male & 382 & $94(24.6)$ & $285(74.6)$ & $3(0.8)$ \\
\hline Female & 140 & $60(42.9)$ & $78(55.7)$ & $2(1.4)$ \\
\hline \multicolumn{5}{|l|}{ Age (years) } \\
\hline $12-19$ & 17 & $10(58.8)$ & $7(41.2)$ & $0(0)$ \\
\hline $20-29$ & 100 & $28(28.0)$ & $71(71.0)$ & $1(1.0)$ \\
\hline $30-39$ & 117 & $33(28.2)$ & $82(70.1)$ & $2(1.7)$ \\
\hline $40-49$ & 132 & 40 (30.3) & 91 (68.9) & $1(0.8)$ \\
\hline $50-59$ & 96 & $28(29.2)$ & $67(69.8)$ & $1(1.0)$ \\
\hline $60-69$ & 45 & $9(20.0)$ & $36(80.0)$ & $0(0)$ \\
\hline$\geq 70$ & 15 & $6(40.0)$ & $9(60.0)$ & $0(0)$ \\
\hline \multicolumn{5}{|l|}{ Weight (kg) } \\
\hline$<50$ & 110 & 100 (90.9) & $6(5.5)$ & $4(3.6)$ \\
\hline $50-70$ & 347 & $42(12.1)$ & 304 (87.6) & $1(0.3)$ \\
\hline$>70$ & 65 & 12 (18.5) & 53 (81.5) & $0(0)$ \\
\hline Total & 522 & $154(29.5)$ & $363(69.5)$ & $5(1.0)$ \\
\hline
\end{tabular}

Table 3. Number and proportion of TB patients treated with different doses of rifampicin in Group II.

\begin{tabular}{|c|c|c|c|c|}
\hline & \multirow{2}{*}{ Patient no. } & \multicolumn{3}{|c|}{ Number (\%) of patients treated with } \\
\hline & & Standard doses & Low doses & High doses \\
\hline \multicolumn{5}{|l|}{ Gender } \\
\hline Male & 145 & $133(91.7)$ & $11(7.6)$ & $1(0.7)$ \\
\hline Female & 46 & $42(91.3)$ & $4(8.7)$ & $0(0)$ \\
\hline \multicolumn{5}{|c|}{ Age (years) } \\
\hline $12-19$ & 3 & $3(100.0)$ & $0(0)$ & $0(0)$ \\
\hline $20-29$ & 39 & 35 (89.7) & $3(7.7)$ & $1(2.6)$ \\
\hline $30-39$ & 44 & 40 (90.9) & $4(9.1)$ & $0(0)$ \\
\hline $40-49$ & 44 & $40(90.9)$ & $4(9.1)$ & $0(0)$ \\
\hline $50-59$ & 43 & $42(97.7)$ & $1(2.3)$ & $0(0)$ \\
\hline $60-69$ & 13 & $11(84.6)$ & $2(15.4)$ & $0(0)$ \\
\hline$\geq 70$ & 5 & $4(80.0)$ & $1(20.0)$ & $0(0)$ \\
\hline \multicolumn{5}{|c|}{ Weight (kg) } \\
\hline$<50$ & 54 & 50 (92.6) & $4(7.4)$ & $0(0)$ \\
\hline $50-70$ & 128 & $118(92.2)$ & $9(7.0)$ & $1(0.8)$ \\
\hline$>70$ & 9 & 7 (77.8) & $2(22.2)$ & $0(0)$ \\
\hline Total & 191 & 175 (91.6) & $15(7.9)$ & $1(0.5)$ \\
\hline
\end{tabular}


Table 4. Number and proportion of TB patients in two Groups with differences in doses of rifampicin received, frequency of rifampicin-resistance and successful outcomes.

\begin{tabular}{|c|c|c|c|}
\hline \multirow{2}{*}{ Variables } & \multicolumn{3}{|c|}{ Patient no. (\%) } \\
\hline & Group $I^{\mathrm{a}}(\mathrm{N}=522)$ & Group $I^{\mathrm{b}}(\mathrm{N}=191)$ & $P$ value \\
\hline Low doses & $363(69.5)$ & $15(7.9)$ & $<0.0001$ \\
\hline Standard doses & $154(29.5)$ & $175(91.6)$ & $<0.0001$ \\
\hline High doses & $5(1.0)$ & $1(0.5)$ & \\
\hline Rifampicin-resistance & $238(45.6)$ & $72(37.7)$ & 0.0304 \\
\hline Successful treatment outcomes & $243(46.6)$ & $105(55.0)$ & 0.0093 \\
\hline
\end{tabular}

${ }^{\mathrm{a} G r o u p ~ I, ~ r i f a m p i c i n ~ o n c e ~ d a i l y ~ t h e r a p y ~ d o s e s ~ r a n g i n g ~ f r o m ~} 300 \mathrm{mg}$ to $750 \mathrm{mg}$; ${ }^{\mathrm{b}}$ Group II, intermittent therapy every other day with combination blister packs of anti-TB drugs containing $600 \mathrm{mg}$ of rifampicin.

resistance rate of isolates collected from patients who received low doses of rifampicin (46.2\% in group I and $40 \%$ in group II) was higher than those collected from patients who received standard and high doses of rifampicin (40.7\% in group I and $37.5 \%$ in group II) within the same treatment group. When treatment outcomes were compared between two groups, we found that $46.6 \%$ of patients (243/522) in treatment group I and $55.0 \%$ of patients (105/191) in treatment group II were successfully treated, and the difference was statistically significant $(P<0.0093)$. The rate of successful treatment for patients in two groups whose isolates were rifampicin-resistance (24.4\% in group I and 34.7\% in group II) was lower than those whose isolates were rifampicin-susceptible within the same treatment group (65.1\% in group I and $67.2 \%$ in group II). Results from this study indicated that the initial treatment for new TB patients with every other day combination blister packs of anti-TB drugs containing rifampicin (group II) was better than those with daily dose of bulk anti-TB drugs including rifampicin (group I) in terms of frequency of rifampicin-resistance and treatment outcomes.

\section{Discussion}

Results from this retrospective multicenter study confirmed that $91.6 \%$ of new TB patients treated at many TB Prevention and Treatment centers (group II), and 29.5\% of those treated at specialized TB hospitals (group I) in China did receive standard doses of rifampicin. Due to less human factors were involved in the use of combination blister packs, most of TB patients in treatment group II received standard doses of rifampicin. However, the use of combination blister packs has its own drawbacks, for example, if patients forget to take the medicines, the time intervals between treatments will be extended which can affect the treatment efficacy. Fixed-dose combination (FDC) tablets containing a variety of anti-TB drugs in the proper dosages were recommended by both WHO and the International Union against Tuberculosis and Lung Diseases (IUATLD) as a means to simplify administration of drugs by reducing the number of pills patients take and decreasing the risks of incorrect prescriptions and development of drug resistance [15]. To prevent the reemergence and spread of MDR-TB, health care providers should follow established guidelines during the entire treatment period for patients with pulmonary TB.

Our results also show that $69.5 \%$ of new TB patients treated at specialized TB hospitals (group I) and $7.9 \%$ of those treated at TB prevention and treatment centers (group II) in multiple provinces of China received low doses of rifampicin. As one of the most important anti-TB drugs, rifampicin must be administered adequately in accordance with the established guidelines [2]-[5] to maximize the cure rate. If the dose is too low, the therapeutic efficacy will be reduced and the risk of drug resistance will be increased [6]. One of the possible reasons for the use of low doses of rifampicin in treatment group I was that physicians at specialized TB hospitals worried about potential adverse reactions of rifampicin, and therefore prescribed low doses of rifampicin based on their personal experience instead of following the established guidelines. We previously found that this kind of practice was quite common at many specialized TB hospitals in China [16] [17]. Similar irrational phenomenon has also occurred in other countries of the world (Kenya, Malawi, Nepal, Senegal, and Chinese Taipei) [18], which is understandable since jaundice hepatitis hepatic necrosis and fatal cases caused by rifampicin were reported in the early literature [19]. In addition, rifampicin could also cause acute allergic reactions, even though the inci- 
dence is very low, and death if patients are not rescued immediately or it happens to elderly patients [20]. Among patients with liver damage caused by anti-TB drugs, 33\% had no obvious symptoms [21], indicating that we need to strengthen the therapeutic drug monitoring (TDM), inform patients and their families in advance about early symptoms of adverse reactions and possible side effects, and prevent adverse reactions through team works [7].

Comparison results show that the percentage of rifampicin-resistance for clinical isolates collected from TB patients in treatment group I (45.6\%) was significantly higher than those in treatment group II (37.7\%), and the rate of successful treatment for patients in treatment group I (46.6\%) was significantly lower than those in treatment group II (55\%). Our results indicate that low doses of rifampicin used in the initial treatment of patients with TB are indeed correlated to increased frequency of rifampicin-resistance and poorer treatment outcomes, and support the idea of using higher doses of rifampicin for new TB patients to shorten the treatment duration, to reduce rifampicin-resistance, and to improve treatment outcomes.

Because this retrospective multicenter study was not a double-blind placebo-controlled clinical trial, it had some potential limitations. In addition to the difference in doses of rifampicin received by TB patients in two treatment groups, other variable factors such as administering methods for rifampicin and doses of three other first-line anti-TB drugs may also affect the frequency of rifampicin-resistance and treatment outcomes. To overcome these potential limitations, confirm the conclusions of the current study, and prove the potential benefits and unanticipated risks of using higher doses of rifampicin, multicenter randomized double-blind placebo-controlled clinical studies with more new patients with pulmonary TB in each group will need to be properly designed and conducted.

Currently, one clinical trial is planning to recruit 180 participants with active, infectious, drug-susceptible TB in Peru to evaluate the potential of higher doses of rifampicin $(15 \mathrm{mg} / \mathrm{kg} /$ day and $20 \mathrm{mg} / \mathrm{kg} / \mathrm{day})$ to shorten treatment for tuberculosis without causing more adverse events (http://clinicaltrials.gov/show/NCT01408914). Results from a recently published study using a murine model indicated that even the standard rifampicin dosage $(10 \mathrm{mg} / \mathrm{kg} / \mathrm{day})$ was too low, and a rifampicin dosage of $80 \mathrm{mg} / \mathrm{kg} /$ day significantly reduced therapy duration without adverse effects, which suggested that much higher doses of rifampicin might lead to a more rapid treatment response and the shortened treatment course [22]. Based on this suggestion, another clinical trial is ongoing in South Africa to determine safety, tolerability, extended early bactericidal activity and pharmaco-kinetics of even higher doses of rifampicin (10, 20, 25, 30, 35, 40, 45, 50 and $55 \mathrm{mg} / \mathrm{kg} /$ day) in adults (18 years to 65 years) with pulmonary TB (http://clinicaltrials.gov/ct2/show/NCT01392911). However, these clinical trials were not designed specifically to determine the correlation between low doses of rifampicin and frequency of rifampicin-resistance as well as treatment outcomes.

\section{Conclusion}

Results from our study indicate that low doses of rifampicin used for the initial treatment of new pulmonary TB patients are correlated to increased frequency of rifampicin-resistance and poorer outcomes, and the difference between two treatment groups is statistically significant. Our results also provide clinical evidence to support the idea of using higher doses of rifampicin to shorten the treatment duration, reduce the frequency of rifampicinresistance, and improve the treatment outcomes for patients with pulmonary TB.

\section{Acknowledgements}

The authors would like to thank all the participants of this study and are greatly appreciated the supports from the following institutions, hospitals, and individuals: Beijing Chest Hospital/Beijing Institute of Tuberculosis and Thoracic Tumor (X Huang), Affiliated Hospital of Zunyi Medical College (JY Zhang), Shenyang Chest Hospital (X Liang), The First Affiliated Hospital of Xinxiang Medical College (H Shang), Heilongjiang Institute of Tuberculosis Control (X Yan), Harbin Chest Hospital (B Ji), the Jingmen Third Hospital of Infectious Diseases (S Chen), Center for Disease Control, Beijing (X Wu), Beijing Geriatric Hospital (W Cao), The Fourth Hospital of Tangshan (P Zhang), the Third Qinhuangdao Hospital (X Leng), Xuzhou Hospital of Infectious Diseases (HQ Zhang), Department of Pneumonoconiosis and Tuberculosis, Beijing Jingmei Group General Hospital (D Chen), The 309th Hospital of Chinese People's Liberation Army (JQ Liang), Anhui Provincial Chest Hospital (GW Chen), Hunan Provincial Tuberculosis Hospital (KY Yang), Infectious Disease Prevention and Treatment Hospital of Heilongjiang Province (YQ Liu), Affiliated Shanghai Pulmonary Hospital of Tongji 
University (SJ Tang), Shandong Provincial Chest Hospital (LH Qiu), Tuberculosis Prevention and Treatment Hospital of Shanxi Province (GQ Niu), The 3rd People's Hospital of Shenzhen City (WY Yu), Shanghai Municipal Center for Disease Prevention and Control (XQ Li), Guangzhou Chest Hospital (SY Tan), Hebei Provincial Center for Disease Prevention and Control (LY Zhang), Center for Disease Prevention and Control of Guangxi Zhuang Autonomous Region (FY Liu), Tuberculosis Prevention and Treatment Hospital of Chongqing City (DY Hu), and Yunnan Provincial Center for Disease Prevention and Control (YL Zhang). This study was funded by the "Eleventh Five-Year" Major National Research Project on treatment strategies for previously treated TB (2008ZX10003-015), Comprehensive Treatment of MDR-TB (2008ZX10003-014), and Clinical Occurrence and Early Warning Model for Drug-Resistant TB (2008ZX10003-008). Study sponsors played no role in study design; in the writing of the report; and in the decision to submit the paper for publication.

\section{Competing Interests}

All authors have completed the ICMJE uniform disclosure form at www.icmje.org/coi disclosure.pdf. All authors except HZ declare no conflicts of interest. HZ has shares in Z-BioMed, which is involved in infectious disease research.

\section{References}

[1] World Health Organization. (2014) Global Tuberculosis Report 2014. http://www.who.int/tb/publications/global_report/en/

[2] World Health Organization. (2010) Guidelines for Treatment of Tuberculosis. 4th Edition. http://www.who.int/tb/publications/2010/9789241547833/en/

[3] Centers for Disease Control and Prevention. Tuberculosis (TB) Treatment. http://www.cdc.gov/tb/topic/treatment/default.htm

[4] Xiao, D.L., Zhao, M.G. and Wang, Y. (2008) Implementation Guidelines for Chinese Tuberculosis Control Programs. Peking Union Medical College Press, Beijing.

[5] Chinese Medical Association. (2005) Guidelines for Clinical Diagnosis and Treatment of Tuberculosis. People’s Health Press, Beijing.

[6] Norval, P.Y. (2010) Review of Prescribing Practices: An Essential Tool for Measuring the Quality of Tuberculosis Services. International Journal of Tuberculosis and Lung Diseases, 14, 795.

[7] Curry International TB Center and California Department of Public Health. (2011) Drug-Resistant Tuberculosis: A Survival Guide for Clinicians. 2nd Edition. http://www.currytbcenter.ucsf.edu/drtb/docs/MDRTB_book_2011.pdf

[8] Nijland, H.M., Ruslami, R., Stalenhoef, J.E., Nelwan, E.J., Alisjahbana, B., Nelwan, R.H., van der Ven, A.J., Danusantoso, H., Aarnoutse, R.E. and van Crevel, R. (2006) Exposure to Rifampicin Is Strongly Reduced in Patients with Tuberculosis and Type 2 Diabetes. Clinical Infectious Diseases, 43, 848-854. http://dx.doi.org/10.1086/507543

[9] Ruslami, R., Nijland, H.M., Adhiarta, I.G., Kariadi, S.H., Alisjahbana, B., Aarnoutse, R.E. and van Crevel, R. (2010) Pharmacokinetics of Antituberculosis Drugs in Pulmonary Tuberculosis Patients with Type 2 Diabetes. Antimicrobial Agents and Chemotherapy, 54, 1068-1074. http://dx.doi.org/10.1128/AAC.00447-09

[10] Ruslami, R., Nijland, H., Aarnoutse, R., Alisjahbana, B., Soeroto, A.Y., Ewalds, S. and van Crevel, R. (2006) Evaluation of High-Versus Standard-Dose Rifampin in Indonesian Patients with Pulmonary Tuberculosis. Antimicrobial Agents and Chemotherapy, 50, 822-823. http://dx.doi.org/10.1128/AAC.50.2.822-823.2006

[11] Hao, L.H., Guo, S.C., Liu, C.C., Zhu, H., Wang, B., Fu, L., Chen, M.T., Zhou, L., Chi, J.Y., Yang, W., Nie, W.J. and Lu, Y. (2014) Comparative Bioavailability of Rifampicin and Isoniazid in Fixed-Dose Combinations and Single-Drug Formulations. International Journal of Tuberculosis and Lung Diseases, 18, 1505-1512. http://dx.doi.org/10.5588/ijtld.13.0647

[12] Zhu, H., Guo, S.C., Hao, L.H., Liu, C.C., Wang, B., Fu, L., Chen, M.T., Zhou, L., Chi, J.Y., Yang, W., Nie, W.J. and Lu, Y. (2015) Relative Bioavailability of Rifampicin in Four Chinese Fixed-Dose Combinations Compared with Rifampicin in Free Combinations. Chinese Medical Journal (English), 128,433-437.

[13] Zhang, Z., Dai, F., Luo, F., Zhong, M., Huang, Z., Hou, T. and Xu, J. (2014) Could High-Concentration Rifampicin Kill Rifampicin-Resistant M. Tuberculosis? Rifampicin MIC Test in Rifampicin-Resistant Isolates from Patients with Osteoarticular Tuberculosis. Journal of Orthopaedic Surgery and Research, 9, 124. http://dx.doi.org/10.1186/s13018-014-0124-1

[14] Technical Guidance Group of the Fifth National TB Epidemiological Survey, Office of the Fifth National TB Epidemiological Survey (2012) 2010 National Tuberculosis Epidemiological Survey in China. Chinese Journal of Antituber- 
culosis, 34, 485-508.

[15] Phanouvong, S., Vrakking, H., Blanc, L., Wondemagegnehu, E., Moore, T., Rägo, L., Mboussa, J., Norval, P. and Matji, R. (2002) Operational Guide for National Tuberculosis Control Programmes on the Introduction and Use of Fixed-Dose Combination Drugs. http://whqlibdoc.who.int/hq/2002/WHO_CDS_TB_2002.308.pdf

[16] Xie, L., Gao, W.W., Bu, J.L. and Ma, Y. (2008) Analysis of 702 Cases of Adverse Drug Reactions Due to Anti-Tuberculosis Drugs. Chinese Journal of Antituberculosis, 30, 275-278.

[17] Xia, Y.Y. and Zhan, S.Y. (2007) Systematic Review of Anti-Tuberculosis Drug Induced Adverse Reactions in China. Chinese Journal of Tuberculosis and Respiratory Diseases, 30, 419-423.

[18] Chiang, C.Y., Bai, K.J., Lee, C.N., Enarson, D.A., Suo, J. and Luh, K.T. (2010) Inconsistent Dosing of Anti-Tuberculosis Drugs in Taipei, Taiwan. International Journal of Tuberculosis and Lung Diseases, 14, 878-883.

[19] Zhang, D.R. (1995) New Concepts of Tuberculosis. Chinese Agricultural Science and Technology Press, Beijing.

[20] Tu, D.H. and Wan, L.Y. (2009) Modern Theories and Practices for Tuberculosis Control. Modern Education Press, Beijing.

[21] Shang, P., Xia, Y., Liu, F., Wang, X., Yuan, Y., Hu, D., Tu, D., Chen, Y., Deng, P., Cheng, S., Zhou, L., Ma, Y., Zhu, L., Gao, W., Wang, H., Chen, D., Yang, L., He, P., Wu, S., Tang, S., Lv, X., Shu, Z., Zhang, Y., Yang, Z., Chen, Y., Li, N., Sun, F., Li, X., He, Y., Garner, P. and Zhan, S. (2011) Incidence, Clinical Features and Impact on Anti-Tuberculosis Treatment of Anti-Tuberculosis Drug Induced Liver Injury (ATLI) in China. PLoS ONE, 6, e21836. http://journals.plos.org/plosone/article?id=10.1371/journal.pone.0021836 http://dx.doi.org/10.1371/journal.pone.0021836

[22] de Steenwinkel, J.E., Aarnoutse, R.E., de Knegt, G.J., ten Kate, M.T., Teulen, M., Verbrugh, H.A., Boeree, M.J., van Soolingen, D. and Bakker-Woudenberg, I.A. (2013) Optimization of the Rifampin Dosage to Improve the Therapeutic Efficacy in Tuberculosis Treatment Using a Murine Model. American Journal of Respiratory and Critical Care Medicine, 187, 1127-1134. http://dx.doi.org/10.1164/rccm.201207-1210OC

\section{List of Abbreviations}

CDC: Centers for Disease Control and Prevention

DST: Drug Susceptibility Testing

FDC: Fixed-Dose Combination

HIV: Human Immunodeficiency Virus

IUATLD: International Union against Tuberculosis and Lung Diseases

L-J: Löwenstein-Jensen

MDR: Multidrug-Resistant

Mtb: Mycobacterium Tuberculosis

TB: Tuberculosis 